\title{
Two-color pump-probe experiments on silicon inverse opals
}

\author{
C. Becker, S. Linden, and G. von Freymann \\ Institut für Nanotechnologie, Forschungszentrum Karlsruhe in der Helmholtz-Gemeinschaft, Postfach 3640, \\ D-76021 Karlsruhe, Germany
}

\author{
M. Wegener \\ Institut für Angewandte Physik, Wolfgang-Gaede-Straße 1, Universität Karlsruhe (TH), D-76131 Karlsruhe, \\ Germany
}

\begin{abstract}
N. Tétreault, E. Vekris, V. Kitaev, and G. A. Ozin
Materials Chemistry Research Group, Department of Chemistry, University of Toronto, 80 St. George Street, Toronto, Ontario M5S 3H6, Canada
\end{abstract}

(Received 5 April 2005; accepted 11 July 2005; published online 24 August 2005)

\begin{abstract}
We present time-resolved pump-probe experiments in a transmission geometry using off-resonant excitation on very high-quality silicon inverse opals. We show that the nonlinear optical response can drastically be modified by tempering of the sample. The as-grown samples are dominated by an absorptive response with recovery times as short as one picosecond. For the tempered samples, both the relaxation and the scattering times increase, leading to a prominent dispersive response. Based on simple calculations using the Drude model, we estimate corresponding refractive index changes as large as $\Delta n=-0.5+\mathrm{i} 0.07$. (c) 2005 American Institute of Physics. [DOI: 10.1063/1.2035873]
\end{abstract}

In recent years, three-dimensional photonic band gap materials have attracted considerable attention. Apart from their linear optical properties, which might serve as a platform for highly integrated photonic circuits, the quantum optical and the nonlinear optical properties ${ }^{1-3}$ are of particular interest. Applications in terms of lasers and all-optical switches, respectively, are envisioned.

For photonic band gap materials in general, theory has predicted interesting ultrafast all-optical switching devices, ${ }^{4-9}$ e.g., based on the optical Kerr effect. The latter results from the real part of the third-order nonlinear optical susceptibility, the corresponding imaginary part is usually not considered. In the simplest case, the photonic band edge provides a sharp spectral feature whose position is shifted by a nonlinear optical index change.

With regard to corresponding experiments, particularly to ultrafast all-optical switching, only few studies have been published. This comprises pump-probe experiments on onedimensional structures, ${ }^{10}$ on two-dimensional photonic bandgap materials, ${ }^{11-14}$ and on three-dimensional photonic crystals without a complete photonic band gap. ${ }^{15-17}$

One vision is to incorporate such nonlinear effects/ switches into highly integrated photonic circuits, which require a complete three-dimensional band gap. Because a complete photonic bandgap requires a large refractive-index contrast, silicon-based structures are of particular interest. In as well as electrochemically etched structures ${ }^{22-24}$ have been discussed. In this letter, we perform femtosecond two-color pump-probe experiments on high-quality silicon inverse opals in transmission geometry.

The linear transmission spectrum of one of our samples, consisting of six layers, is shown in Fig. 1. The sample has been fabricated with a low-pressure self-assembly approach using $860 \mathrm{~nm}$ diameter silica spheres ${ }^{25}$ followed by infiltration with silicon via a chemical vapor deposition (CVD) process. ${ }^{20}$ Subsequently, the silica template as well as the recent years, inverse opals, ${ }^{18-20}$ layer-by-layer structures, ${ }^{21}$ glass substrate are removed by wet chemical etching, leading

to a freestanding silicon inverse opal (inset). The latter is crucial because we have found in a number of independent experiments that the nonlinear optical contribution of a substrate (e.g., glass) can easily exceed that of the photonic band gap material, leading to undesired artifacts. The silicon grown by CVD is known to be amorphous. Subsequent tempering can bring it into a polycrystalline state. Note that the fundamental photonic stop band in Fig. 1 is well developed and that the transmission energetically above the stop band is as large as $65 \%$. Tempering for one day at $600{ }^{\circ} \mathrm{C}$ reduces the silicon refractive index from 3.95 to 3.48 , hence shifting the stop band towards the blue.

In our pump-probe experiments, we use two optical parametric oscillators (OPA) that can be tuned independently from 1.2 to $2.4 \mu \mathrm{m}$, both pumped by a regeneratively amplified Ti:sappire laser system (Spectra-Physics Hurricane). Pump and probe have durations of about $130 \mathrm{fs}$, have the same linear polarization, and are independently focused with

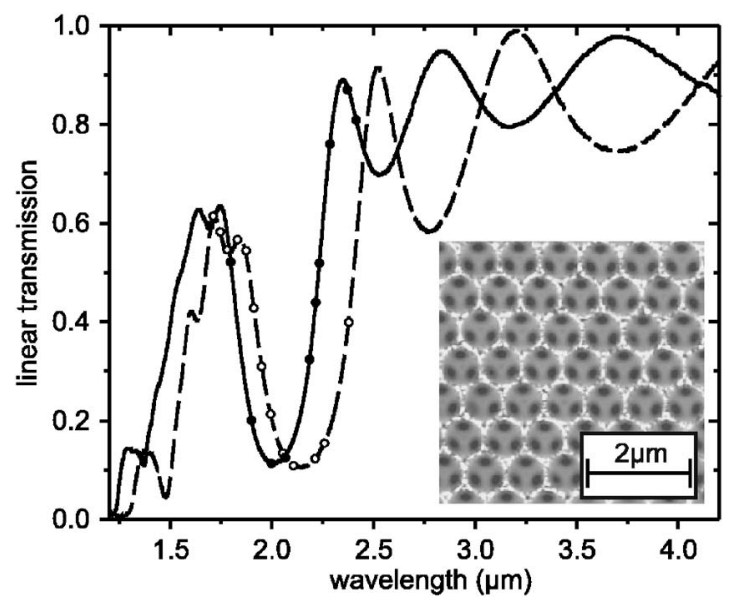

FIG. 1. Linear transmission spectra of the silicon inverse opal in [111] direction, as-grown (dashed) and tempered (solid). The dots correspond to the probe wavelengths investigated in Fig. 2. The inset shows an SEM image of the sample surface.

EVA-STAR (Elektronisches Volltextarchiv - Scientific Articles Repository)

0003-6951/2005/87(9)/091111/3/\$22.50 http://digbib.ubka.uni-karlsruhe.de/volltexte/1000010955

(C) 2005 American Institute of Physics

Downloaded 18 Mar 2009 to 129.13.72.198. Redistribution subject to AIP license or copyright; see http://apl.aip.org/apl/copyright.jsp 


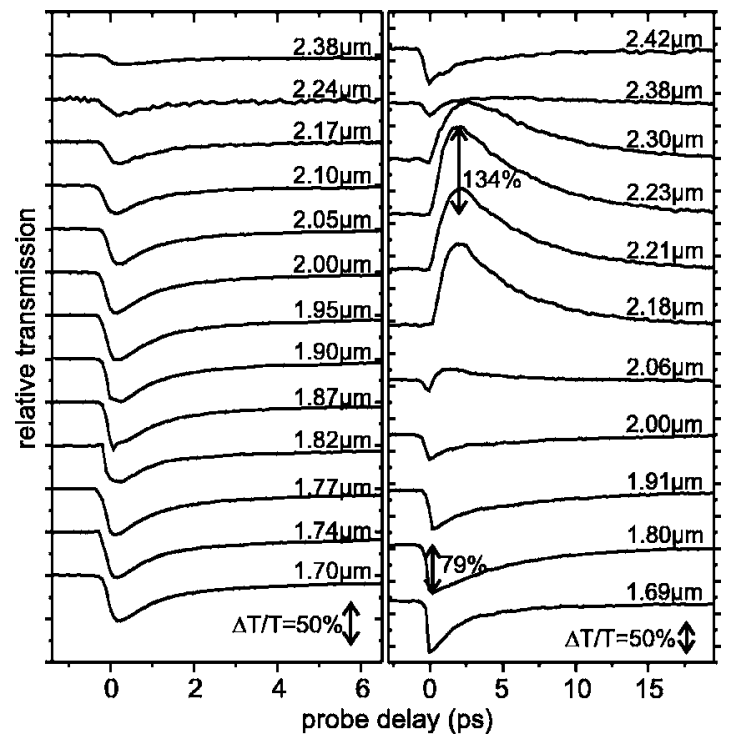

FIG. 2. Pump-probe traces for several probe wavelengths (as indicated), vertically displaced for clarity. LHS column: As-grown sample (a-Si), $\lambda_{\text {pump }}=1.84 \mu \mathrm{m}, I_{\text {pump }}=66 \mathrm{GW} / \mathrm{cm}^{2}$; RHS column: Tempered sample (polycrystalline $\mathrm{Si}$ ), $\lambda_{\text {pump }}=1.73 \mu \mathrm{m}, I_{\text {pump }}=90 \mathrm{GW} / \mathrm{cm}^{2}$. All curves in one column are shown on the same scale of transmission relative to the unexcited sample (see arrows). Note the different time scales on the LHS and RHS, respectively.

different lenses to allow for a larger pump spot size on the sample to ensure homogeneous excitation conditions. We have found, however, that the spot sizes strongly depend on wavelength. In the best case, the pump spot is six times larger than the probe, in the worst case three times, corresponding to a pump/probe intensity ratio of $I_{\text {pump }} / I_{\text {probe }}=30$. This dependence favors sets of experiments in which we tune the probe wavelength $\lambda_{\text {probe }}$ for fixed pump wavelength $\lambda_{\text {pump. }}$. Furthermore, we focus on excitation well below the electronic silicon band gap (actually, the amorphous silicon mobility edge), for which theory has discussed the instantaneous optical Kerr effect, as discussed above.

The LHS column of Fig. 2 shows a series of time traces for the as-grown sample (i.e., untempered) for fixed pump wavelength, fixed pump intensity, and varying probe wavelength as indicated. "Relative transmission" refers to the transmission with respect to the linear optical case. The RHS column shows corresponding results after tempering. Obviously, the nonlinear responses are completely different. For the untempered sample (LHS), we find induced absorption for all wavelengths and a recovery time of just $1 \mathrm{ps}$. In contrast, after tempering (RHS), the recovery times increase to about 5 ps and a shift of the fundamental stop band is observed: On the long-wavelength (short-wavelength) side of the stop band, we find increased (reduced) transmission - as expected for a reduction of the silicon refractive index after excitation with the pump. Such index reduction is expected from free carriers, which are generated via two-photon absorption. Indeed, in additional experiments, we find that the transmission change scales quadratically with the pump fluence (not shown).

For the interpretation of the data it is instructive to recall some well-known numbers for amorphous and polycrystalline silicon, respectively. Carrier recombination times in the range of just a few picoseconds are quite typical for amorphous ${ }^{26}$ or for radiation damaged silicon. ${ }^{27}$ These numbers are consistent with the short decay times apparent from Downloaded 18 Mar 2009 to 129.13.72.198. Redistribution subject

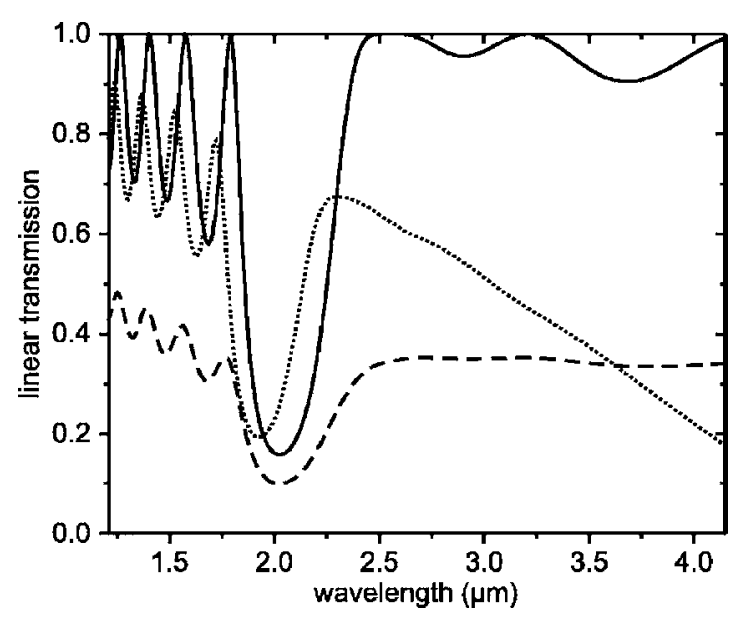

FIG. 3. Calculated transmission for three different parameter sets. The solid curve shows the linear spectrum (i.e., $n_{\mathrm{e}}=0$ ). The other two curves correspond to an optically excited electron density of $n_{\mathrm{e}}=1.5 \times 10^{20} \mathrm{~cm}^{-3}$ and $\tau$ $=0.5 \mathrm{fs}$ (dashed curve) and $\tau=10 \mathrm{fs}$ (dotted curve), respectively.

the LHS of Fig. 2. Furthermore, random variations of the microscopic potential break momentum-conservation restrictions for carrier scattering, leading to electron scattering times in amorphous silicon even below one femtosecond. ${ }^{28}$ This causes a broad and featureless nonlinear optical response and leads to a dominance of absorptive rather than dispersive effects. In crystalline silicon, much longer scattering times are expected.

To further analyze and interpret our experimental data, we have performed simple model calculations based on the scalar wave approximation. ${ }^{29} \mathrm{We}$ assume that $50 \%$ of the voids in the opal template are filled with silicon and consider six layers. The dielectric function $\epsilon(\omega)$ of silicon at frequency $\omega$ is taken as the sum of a constant term and the Drude free-carrier contribution, i.e., $\quad \epsilon(\omega)=(3.48)^{2}$ $-n_{e} e^{2} /\left(m_{\text {eff }} \epsilon_{0}\right)\left(1 /\left(\omega^{2}+\tau^{-2}\right)+\mathrm{i}\left(\tau^{-1} /\left(\omega^{3}+\omega \tau^{-2}\right)\right)\right)$. Here, $n_{\mathrm{e}}$ is the optically excited electron density, e the elementary charge, $\tau$ the Drude scattering time, and $m_{\text {eff }}$ the effective electron mass. The latter is chosen to be $20 \%$ of the free electron mass. The calculated linear transmission spectrum as well as two nonlinear spectra are depicted in Fig. 3. The linear spectrum (solid curve) qualitatively resembles the measured transmission spectrum (Fig. 1). The shape of the nonlinear spectrum sensitively depends on the choice of $\tau$. For $\tau=0.5$ fs (dashed curve in Fig. 3), the calculated spectrum is dominated by a broad and featureless increase of absorption without any major stop-band shift (also compare LHS of Fig. 2). In contrast, for $\tau=10 \mathrm{fs}$ (dotted curve in Fig. 3), a clear blueshift of the fundamental stop band results (also compare RHS of Fig. 2), which corresponds to a calculated maximum change in refractive index of $\Delta n=-0.5$ $+\mathrm{i} 0.07$ at $2.2 \mu \mathrm{m}$ wavelength. For comparison: For the optical Kerr effect with $n_{2}=4.3 \times 10^{-14} \mathrm{~cm}^{2} / \mathrm{W}$ for silicon, ${ }^{30}$ one would expect $\Delta n=n_{2} I_{\text {pump }}=+3.9 \times 10^{-3}$ under our conditions.

In conclusion, we have systematically studied the nonlinear optical response of state-of-the-art silicon inverse opals which can serve as a model system for threedimensional silicon-based photonic band gap materials. We have shown that the nonlinear optical response dramatically depends on the underlying electron scattering times. The latter can be tailored by sample tempering, initiating a transforto AIP license or copyright; see http://apl.aip.org/apl/copyright.jsp 
mation from amorphous to polycrystalline silicon. We achieve nonlinear optical transmission changes as large as $134 \%$ or stop band shifts on the order of $150 \mathrm{~nm}$, corresponding to estimated refractive index changes of $\Delta n=$ $-0.5+\mathrm{i} 0.07$. This significantly surpasses all previously measured effects on three-dimensional photonic crystals. At these excitation levels, two-photon absorption (related to the imaginary part of the third-order nonlinear susceptibility) completely dominates over the nonlinear refractive index $n_{2}$ (related to the real part of the third-order nonlinear susceptibility), which has previously mostly been employed in the theoretical description of the nonlinear optical properties of photonic crystals.

The authors acknowledge support by the Deutsche Forschungsgemeinschaft through Subproject A1.4 of the DFG-Forschungszentrum "Functional Nanostructures" $(\mathrm{CFN})$. The research of M. W. is further supported by DFG Project We 1497/9-1.

${ }^{1}$ N. A. R. Bhat and J. E. Sipe, Phys. Rev. E 64, 056604 (2001).

${ }^{2}$ Y.-K. Ha, J.-E. Kim, H. Y. Park, C.-S. Kee, and H. Lim, Phys. Rev. B 66, 075109 (2002).

${ }^{3}$ P. M. Johnson, A. F. Koenderink, and W. L. Vos, Phys. Rev. B 66, 081102 (2002).

${ }^{4}$ M. Scalora, J. P. Dowling, C. M. Bowden, and M. J. Bloemer, Phys. Rev. Lett. 73, 1368 (1994).

${ }^{5}$ P. Tran, Opt. Lett. 21, 1138 (1996).

${ }^{6}$ M. Soljacic, M. Ibanescu, S. G. Johnson, Y. Fink, and J. D. Joannopoulos, Phys. Rev. E 66, 055601 (2002).

${ }^{7}$ M. Yanik, S. Fan, M. Soljacic, and J. D. Joannopoulos, Opt. Lett. 28, 2506 (2003).

${ }^{8}$ M. Florescu and S. John, Phys. Rev. A 69, 053810 (2004).

${ }^{9}$ R. Z. Wang and S. John, Phys. Rev. A 70, 043805 (2004).

${ }^{10}$ A. Haché and M. Bourgeois, Appl. Phys. Lett. 77, 4089 (2000).

${ }^{11}$ S. W. Leonard, H. M. van Driel, J. Schilling, and R. B. Wehrspohn, Phys. Rev. B 66, 161102 (2002).
${ }^{12}$ A. Bristow, J.-P. Wells, W. Fan, A. Fox, M. Skolnick, D. Whittaker, A. Tahraoui, T. Krauss, and J. Roberts, Appl. Phys. Lett. 83, 851 (2003).

${ }^{13}$ H. W. Tan, H. M. van Driel, S. L. Schweizer, R. B. Wehrspohn, and U. Gösele, Phys. Rev. B 70, 205110 (2004).

${ }^{14}$ X. Hu, Y. Liu, J. Tian, B. Cheng, and D. Zhang, Appl. Phys. Lett. 86, $121102(2005)$.

${ }^{15}$ X. Hu, Q. Zhang, Y. Liu, B. Cheng, and D. Zhang, Appl. Phys. Lett. 83, 2518 (2003).

${ }^{16}$ D. Mazurenko, R. Kerst, J. Dijkhuis, A. Akimov, V. Golubev, D. Kurdyukov, A. Pevstov, and A. Sel'kin, Phys. Rev. Lett. 91, 213903 (2003).

${ }^{17}$ D. Mazurenko, R. Kerst, J. Dijkhuis, A. Akimov, V. Golubev, A. Kaplyanskii, D. Kurdyukov, and A. Pevtsov Appl. Phys. Lett. 86, 041114 (2005).

${ }^{18}$ A. Blanco, E. Chomski, S. Grabtchak, M. Ibisate, S. John, S. W. Leonard, C. Lopez, F. Meseguer, H. Miguez, J. P. Mondia, G. A. Ozin, O. Toader, and H. M. van Driel, Nature (London) 405, 437 (2000).

${ }^{19}$ Y. A. Vlasov, X.-Z. Bo, J. C. Sturm, and D. J. Norris, Nature (London) 414, 289 (2001).

${ }^{20}$ N. Tétreault, H. Miguez, and G. A. Ozin, Adv. Mater. (Weinheim, Ger.) 16, 1471 (2004).

${ }^{21}$ S. Y. Lin, J. G. Fleming, D. L. Hetherington, B. K. Smith, R. Biwas, K. M. Ho, M. M. Sigalas, W. Zubrzycki, S. R. Kurtz, and J. Bur, Nature (London) 394, 251 (1998).

${ }^{22}$ A. Birner, R. B. Wehrspohn, U. M. Gösele, and K. Busch, Adv. Mater. (Weinheim, Ger.) 13, 377 (2001).

${ }^{23}$ S. Matthias, F. Müller, C. Jamois, R. B. Wehrspohn, and U. Gösele, Adv. Mater. (Weinheim, Ger.) 16, 2167 (2004).

${ }^{24}$ J. Schilling, J. White, A. Scherer, G. Stupian, R. Hillebrand, and U. Gösele, Appl. Phys. Lett. 86, 011101 (2005).

${ }^{25}$ V. Kitaev and G. A. Ozin, Adv. Mater. (Weinheim, Ger.) 15, 75 (2003).

${ }^{26}$ A. Mourchid, D. Hulin, R. Vanderhaghen, and P. M. Fauchet, Semicond. Sci. Technol. 7, B302 (1992).

${ }^{27}$ K. P. H. Lui and F. A. Hegmann, Appl. Phys. Lett. 78, 3478 (2001).

${ }^{28}$ P. M. Fauchet, D. Hulin, R. Vaderhaghen, A. Mourchid, and W. L. Nighan, Jr., J. Non-Cryst. Solids 141, 76 (1992).

${ }^{29}$ D. M. Mittleman, J. F. Bertone, P. Jiang, K. S. Hwang, and V. L. Colvin, J. Chem. Phys. 111, 345 (1999).

${ }^{30}$ M. Dinu, F. Quochi, and H. Garcia, Appl. Phys. Lett. 82, 2954 (2003). 